\title{
Conjoint Nerve Root an Intraoperative Challenge in Minimally Invasive Tubular Discectomy
}

\author{
Ayush Sharma, Vijay Singh, Romit Agrawal, Nilesh Mangale, Priyank Deepak, Jeet Savla, Ajay Jaiswal \\ Department of Orthopedic and Spine Surgery, Dr. Babasaheb Ambedkar Central Railway Hospital, Mumbai, India
}

Conjoint nerve root (CNR) is an embryological nerve root anomaly that mainly involves the lumbosacral region. The presence of CNR during tubular discectomy raises the chances of failure in spinal surgery and the risk of neural injuries. Tubular discectomy can be challenging in the presence of CNR owing to limited visualization. Here, we present a technical note on two cases of L5-S1 disc prolapse in the presence of conjoint S1 nerve root that was operated via a minimally invasive tubular approach. Any intraoperative suspicion of CNR while using the tubular approach should prompt the surgeon to perform a thorough tubular decompression prior to nerve root retraction. In patients with a large disc, disc should be approached via the axilla because the axillary area between the dura and the medial boarder of the root is very easy to approach in the presence of CNR. Safe performance of tubular discectomy is possible even in the presence of CNR in the lumbar spine.

Keywords: Conjoined lumbosacral nerve roots; Lumbar disc herniation; Nerve root anomalies; Tubular discectomy

\section{Introduction}

Conjoint nerve root (CNR) is embryological nerve root anomaly that is most commonly observed in the lumbosacral region. The incidence of CNR varies from $2 \%$ to $17.3 \%$ [1-3]. Undiagnosed CNR has been associated with surgical failure [4]. Moreover, simple discectomy without adequate decompression is associated with failed lumbar surgeries in the CNR [5]. Incidental nerve root injuries may occur if the additional nerve root is not visualized during these surgeries. Minimally invasive (MIS) tubular discectomy is becoming a popular option in lumbar spine surgery. MIS offers the advantage of small incision and faster rehabilitation at the cost of limited visualization.
MIS tubular discectomy for CNR is challenging because of the limited visualization and added risk of incidental nerve root injuries. To our knowledge, this is the first report of successful tubular discectomy in the presence of CNR.

\section{Technical Note}

\section{Case 1}

1) Patient

A 64-year-old man presented with left-sided lumbar radiculopathy that had persisted for 3 months; the severity of his symptoms had increased in the previous 20 days.

Received May 19, 2020; Revised Jul 13, 2020; Accepted Jul 13, 2020

Corresponding author: Ayush Sharm

Department of Orthopedic and Spine Surgery, Dr. Babasaheb Ambedkar Central Railway Hospital, Mumbai, India

Tel: +91-9004549623, Fax: +91-2267452360, E-mail: drayushsharma@gmail.com 
His clinical examination revealed sensory deficit in left S1 dermatome. Magnetic resonance imaging (MRI) confirmed broad left paracentral disc prolapse at L5-S1 compressing the left S1 nerve root and the left-sided thecal sac (Fig. 1). The patient was scheduled to undergo left-sided tubular discectomy.

\section{2) Technical note}

After using a routine tubular approach, the surgeon observed that the dura and the S1 nerve root were unusually tense and difficult to retract safely for performing discectomy. The MRI had shown a moderate paracentral disc prolapse; therefore, this was an unusual finding considering the level L5-S1. This prompted to undercutting of the facets using a high-speed burr to create more space for retraction. Undercutting of the facets revealed the pres-

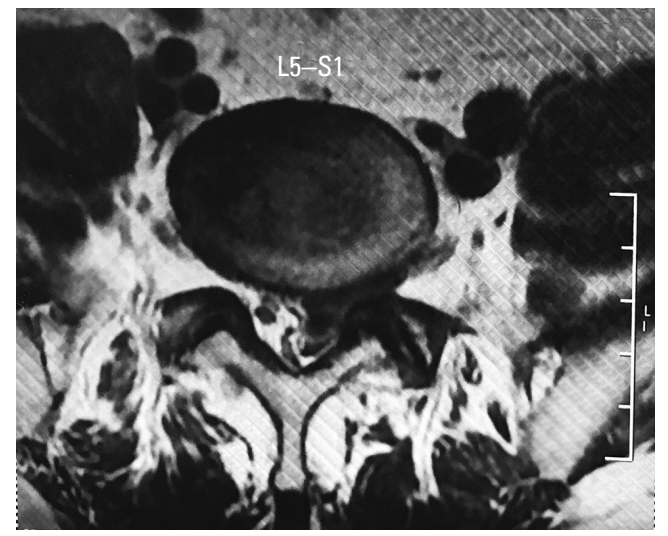

Fig. 1. Magnetic resonance imaging showing broad left paracentral disc at L5S1 compressing left S1 nerve root and left sided thecal sac.

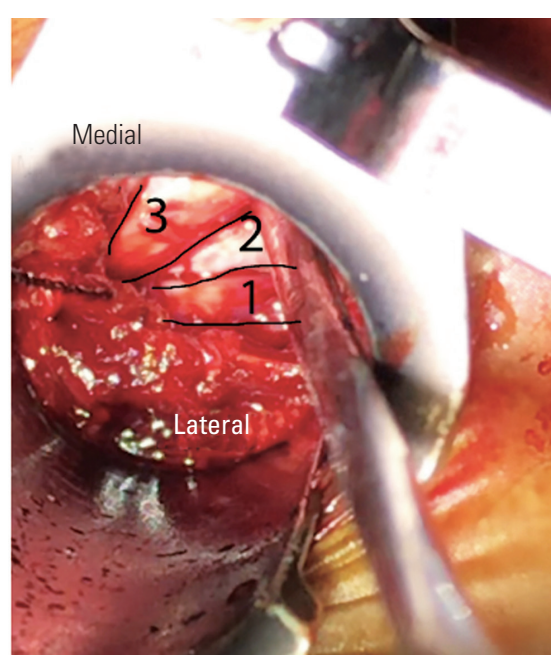

Fig. 2. Intraoperative image showing conjoint nerve root after undercutting ipsilateral left facet. Conjoint roots are marked as $1 \& 2$, and dura is marked as 3 . ence of CNR (Fig. 2). Due to the overcrowding of neural structures the retraction of nerve roots was difficult, so it was decided to perform over the top decompression of the dura and contralateral side. The tubes were tilted to the contralateral side. The ligamentum flavum was removed, the right lateral facets were undercut, and the decompression was continued until the contralateral S1 root was completely free and mobile (Fig. 3). This created enough space for the CNR and dura to be retracted safely. An extruded disc was removed after retracting the CNR and dura to the contralateral side. Mobility of the CNR and dura was confirmed after the discectomy. Adequate decompression and burring of the contralateral facets allowed enough space for safe retraction of the CNR in this case. Patient had good symptomatic relief after the surgery. The Visual Analog Scale (VAS) score improved from 7 to 3 and the Oswestry Disability Index (ODI) improved from 46 to 18. Postoperative magnetic resonance (MR) neurography confirmed type la nerve root sleeve of the left S1 and S2 nerve roots (Fig. 4).

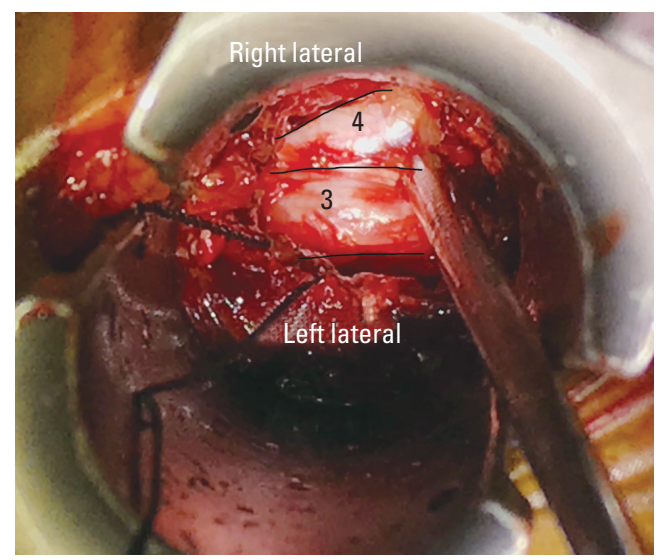

Fig. 3. Intraoperative image showing successful over the top decompression of contralateral side. Dura is marked as 3 , and contralateral left $\mathrm{S} 1$ root is marked as 4 .

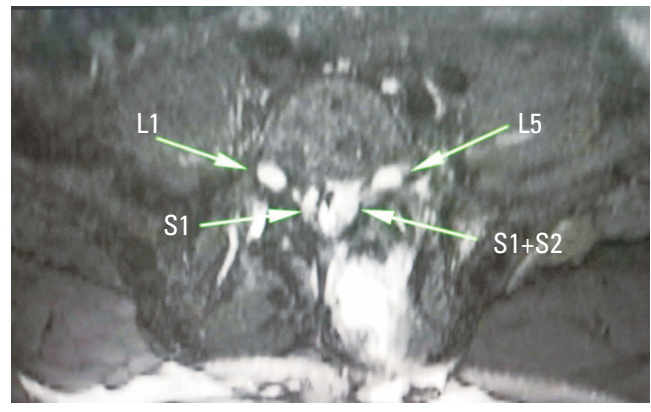

Fig. 4. Postoperative magnetic resonance neurography confirming type 1a nerve root sleeve of the left S1 and S2 roots. 
The patient has consented to the submission of the case report for submission to the journal.

\section{Case 2}

\section{1) Patient}

A 40-year-old woman presented with left-sided radiculopathy and neurological claudication for 3 months. The patient had a VAS of 9 and an ODI of 34 with claudication distance of $50 \mathrm{~m}$. MRI showed left paracentral herniation at L5-S1 that compressed the left S1 root and left-sided thecal sac. After failed conservative treatment, the patient was scheduled for surgery with a left-sided tubular approach.

\section{2) Technical note}

Similar to that in the previous case, intraoperative undercutting of the left facet joints revealed the presence of conjoint roots (Fig. 5, Supplemental Video 1). Based on our previous experience, we first decided to add a contralateral over-the-top decompression; thereafter, the contralateral facets were under cut. Once the dura was completely decompressed, the cord was retracted to the contralateral side. However, unlike the above-mentioned case, in case 2, the CNR was still very tight owing to the underlying compression of the prolapsed disc. It was not possible to retract the CNR and the dura to approach the disc from the shoulder. Therefore, we first approached the disc via the axillary side (between the dura and medial boarder of the root) following the retraction of the dura to contralateral side (Fig. 6). After removing the axillary

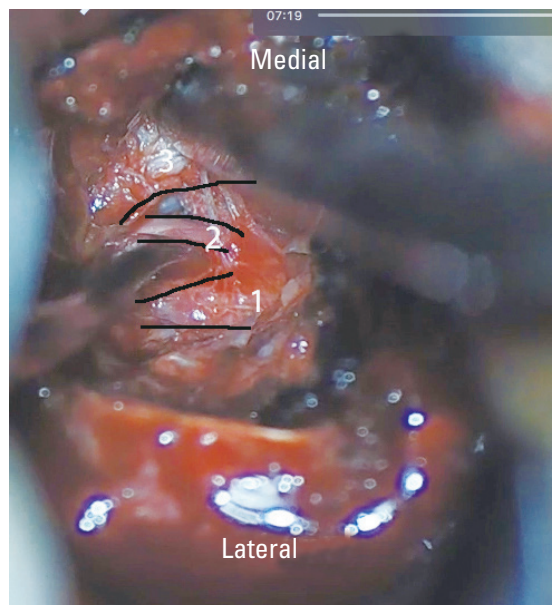

Fig. 5. Intraoperative image showing conjoint nerve root after undercutting ipsilateral left facet. Conjoint roots are marked as $1 \& 2$, and dura is marked as 3 . disc, the conjoint roots were relaxed enough to approach the disc prolapse through the shoulder area (Fig. 7). The post-surgery patient VAS improved to 2, and the ODI improved to 16. Postoperative MR confirmed type 1a S1-S2 CNR.

The patient has consented to the submission of the case report for submission to the journal.

\section{Discussion}

Preoperative identification of CNR is based on a high degree of suspicion. The presence of neurological claudication with radiculopathy in a setting of a relatively small disc compression should raise the suspicion of CNR [4]. With advances in diagnostic modalities, there are increasing reports of CNR. MRI-based diagnosis of CNR is based on indirect imaging signs, such as asymmetry of dura (corner sign), atypical extradural fat (fat crescent sign), or

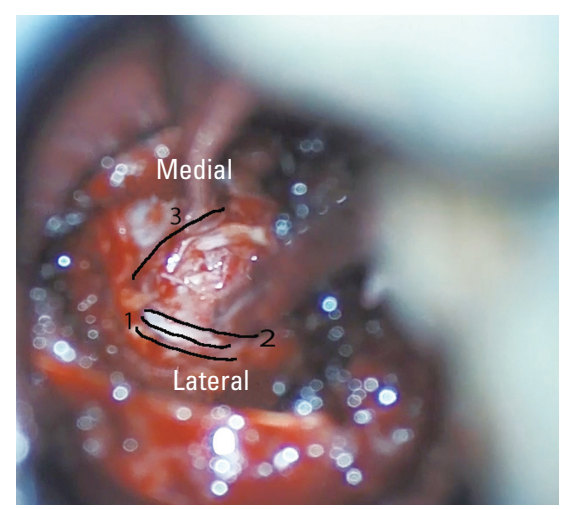

Fig. 6. Intraoperative image showing disc being approached through the axilla between the conjoint roots and dura. Conjoint roots are marked as $1 \& 2$, and dura is marked as 3 .

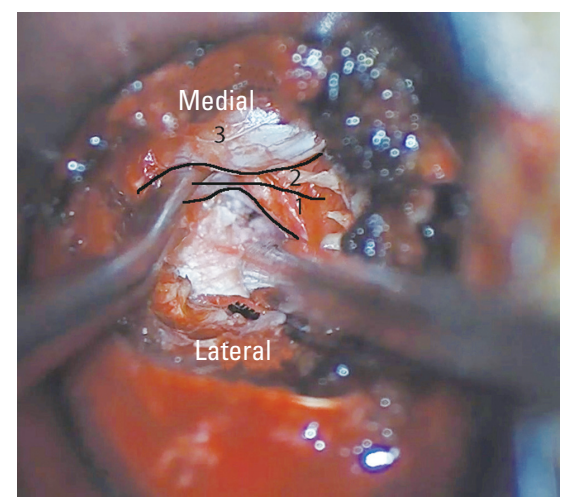

Fig. 7. Intraoperative image showing disc being approached through the shoulder after retracting the conjoint roots medially. Conjoint roots are marked as 1 $\& 2$, and dura is marked as 3 . 
angulation of existing nerve root (parallel sign) [6]. These indirect signs are not very specific and can be mimicked by disc herniation or extradural lesions. Newer diagnostic tools, such as MR neurography, can directly demonstrate CNRs [7]. MR neurography allows direct and more accurate diagnosis of neural variations and CNRs. With advances in MIS surgery, the tubular approach is rapidly becoming a popular method for discectomy. The MIS approach for spine surgeries uses a small surgical incision and less extensive muscle dissection, causing less postoperative pain, earlier discharge, and more rapid return to normal activities [8-10]. Tubular discectomy offers certain advantages; however, it has certain drawbacks, such as limited tubular visualization. Attempting a tubular discectomy in presence of CNR can be challenging because it raises the risk of neural injuries [11]. In the setting of disc prolapse with CNR, the presence of an additional nerve root further compromises an already compromised dural space. The tethering effect of CNRs and large crosssectional area of abnormal nerve root make these surgeries more challenging with the additional risk of iatrogenic injury [12]. The key to successful surgery here is adequate removal of the prolapsed disc fragment and wise decompression of neural elements. These principles of surgery should be applied while attempting to use the tubular approach in CNR patients. After using a routine tubular approach, it is advised to burr or undercut the ipsilateral facet joint for complete visualization of the lateral most edge of the nerve roots. If root retraction is still difficult, the surgeon can attempt an over-the-top decompression of the dura. Once the dura is adequately mobile, the CNR could be retracted to the contralateral side along with the dura to safely remove the disc fragments. In cases with a large disc, the recommendation is to approach the disc through the axilla because the axillary area between the dura and the medial border of the root is easy to approach in the presence of CNR. Once the axillary disc is removed, the CNR can be mobilized medially to approach the disc through the shoulder area. Adequate decompression is crucial for the achievement of good surgical outcome in cases of tubular discectomy in the presence of CNR.

In conclusion, the presence of CNR during tubular discectomy can be challenging owing to limited visualization in the MIS approach. Any intraoperative suspicion should prompt the surgeon to perform thorough decompression before attempting nerve root retraction in patients with CNR. In patients with a large disc, the recommendation is to approach the disc through the axilla because the axillary area between the dura and medial boarder of the root is easier to approach in the presence of CNR.

\section{Conflict of Interest}

No potential conflict of interest relevant to this article was reported.

\section{Supplementary Materials}

Supplementary materials can be available from https:// doi.org/10.31616/asj.2020.0250.v001. Supplemental Video 1. Intraoperative video showing steps of tubular approach, decompression, and discectomy for case 2 .

\section{References}

1. Aota Y, Saito Y, Yoshikawa K, Asada T, Kondo S, Watanabe K. Presurgical identification of extradural nerve root anomalies by coronal fat-suppressed magnetic resonance imaging: a report of six cases and a review of the literature. J Spinal Disord 1997;10:16775.

2. Artico M, Carloia S, Piacentini M, et al. Conjoined lumbosacral nerve roots: observations on three cases and review of the literature. Neurocirugia (Astur) 2006;17:54-9.

3. Broom MJ. Congenital anomalies of the lumbosacral spine causing nerve root entrapment: the role of high resolution CT in diagnosis. Orthopedics 1994;17:637.

4. Lotan R, Al-Rashdi A, Yee A, Finkelstein J. Clinical features of conjoined lumbosacral nerve roots versus lumbar intervertebral disc herniations. Eur Spine J 2010;19:1094-8.

5. Scuderi GJ, Vaccaro AR, Brusovanik GV, Kwon BK, Berta SC. Conjoined lumbar nerve roots: a frequently underappreciated congenital abnormality. J Spinal Disord Tech 2004;17:86-93.

6. Trimba R, Spivak JM, Bendo JA. Conjoined nerve roots of the lumbar spine. Spine J 2012;12:515-24.

7. Engar C, Wadhwa V, Weinberg B, Chhabra A. Conjoined lumbosacral nerve roots: direct demonstration on MR neurography. Clin Imaging 2014;38:892-4.

8. Lee P, Liu JC, Fessler RG. Perioperative results following open and minimally invasive single-level 
lumbar discectomy. J Clin Neurosci 2011;18:1667-70.

9. Smith ZA, Fessler RG. Paradigm changes in spine surgery: evolution of minimally invasive techniques. Nat Rev Neurol 2012;8:443-50.

10. Wong AP, Smith ZA, Lall RR, Bresnahan LE, Fessler RG. The microendoscopic decompression of lumbar stenosis: a review of the current literature and clinical results. Minim Invasive Surg 2012;2012:325095.
11. Webb J, Gottschalk L, Lee YP, Garfin S, Kim C. Surgeon perceptions of minimally invasive spine surgery. SAS J 2008;2:145.

12. Burke SM, Safain MG, Kryzanski J, Riesenburger RI. Nerve root anomalies: implications for transforaminal lumbar interbody fusion surgery and a review of the Neidre and Macnab classification system. Neurosurg Focus 2013;35:E9. 